\title{
Limitaciones tecnológicas que afectan la gestión del sistema productivo litográfico en Tunja
}

\author{
Technological limitations affecting management of \\ lithographic production system in Tunja
}

\section{GLORIA ELIZABETH GRIMALDO LEÓN}

Ingeniera de Producción Industrial

Mg. Administración y Dirección de Empresas

Grupo de Investigación LOGyCA Universidad de Boyacá, Colombia gegrimaldo@uniboyaca.edu.co

\section{SONIA MILENA FORERO ROPERO}

Ingeniera Industrial

Mg. Dirección Estratégica Grupo de Investigación LOGyCA Universidad de Boyacá, Colombia sonforero@uniboyaca.edu.co

\section{EDUIN D. CONTRERAS CASTAÑEDA}

Ingeniero Industrial Mg. Diseño y Gestión de Procesos Grupo de Investigación LOGyCA Universidad de Boyacá, Colombia econtreras@uniboyaca.edu.co 


\title{
RESUMEN
}

Se presentan los resultados de la primera fase del proyecto titulado: "Herramientas de mejora continua, aplicadas al sector litográfico de Tunja”, planteado con el propósito de identificar la situación actual de los principales elementos que conforman el proceso de producción y a su vez, describir la gestión del sistema productivo desde la perspectiva del empresario, enfatizando en los factores tecnológicos. Se empleó una metodología de estudio de caso, la información se recolectó mediante encuesta dirigida a los gerentes de los procesos. Los principales hallazgos relacionados con las limitaciones tecnológicas se presentaron en la aplicación de software para la planeación, programación y control de producción, antigüedad de maquinaria y equipos, deficiencia en la gestión del mantenimiento y desconocimiento de herramientas gerenciales para la adecuada gestión tecnológica de la empresa. Por lo tanto, se puede considerar que el sistema de producción del sector litográfico posee limitaciones tecnológicas que obstaculizan su crecimiento en un mercado competitivo global.

Palabras Clave: Gestión tecnológica, sistema productivo, autodiagnóstico, industria litográfica.

\begin{abstract}
This paper discloses the results of the first phase of the project entitled: "continuous improvement tools, applied to the lithographic sector of Tunja", formulated for the goal of identifying the actual state of the main elements of the production process, as well as describe the production system management from the perspective of the manager, emphasizing technological factors. Using a case study methodology, information was collected by survey of managers of processes. The main findings related to technological limitations occurred in the software application for planning, scheduling and production control, age of machinery and equipment, deficient maintenance management and lack of awareness of technology management tools. Therefore, the lithographic production system has technological limitations that hinder its growth in a competitive global market.
\end{abstract}

Keywords: Technology management, production system, self-assessment, lithographic industry. 


\section{INTRODUCCIÓN}

La actividad económica de Tunja se fundamenta en el comercio y el turismo, la ciudad presenta como sectores predominantes la intermediación financiera, el comercio y el transporte. Por su parte, la dinámica productiva está representada por instituciones de educación, instituciones prestadoras de servicios de salud, empresas promotoras de salud y establecimientos financieros. Para lograr un verdadero desarrollo industrial, es necesario considerar las pequeñas y medianas industrias que proveen los bienes y servicios necesarios para el adecuado funcionamiento de las actividades productivas descritas. De allí, parte la necesidad de identificar al sector litográfico como un potencial industrial de la ciudad.

Según información de la Gobernación de Boyacá, el sector litográfico de Tunja está compuesto por ocho empresas (Alcaldía de Tunja, 2010), pero si se observa la realidad de la ciudad se pueden encontrar alrededor de 50 establecimientos dedicados a labores de impresión, tipografía y afines (Cámara de Comercio de Tunja, 2010). Según información suministrada por la Cámara de Comercio de Tunja, el sector litográfico está compuesto por pequeñas y medianas industrias, puesto que emplean menos de 50 personas y no cuentan con el capital suficiente para ser catalogadas en otra clasificación, teniendo en cuenta lo estipulado en el artículo 2 de la Ley 905 de 2004 del Congreso de Colombia (2004), convirtiendo esta actividad industrial en un oficio artesanal dada la pobre infraestructura y capacidad de las empresas.

A su vez, es importante resaltar que las habilidades y técnicas administrativas de la industria gráfica han cambiado poco en los últimos 5 años. Los empresarios creen que la producción de impresos es un arte, un oficio y que para optimizar sus operaciones deben tener equipos más rápidos y tecnologías más modernas. Sin embargo, no es realista la práctica de comprar equipos nuevos como método para solucionar problemas.

Basados en lo anterior, en el presente artículo se exponen los resultados de la revisión al sistema productivo litográfico, con miras a diseñar e implementar estrategias de mejora continua en sus procesos. Los resultados se dan a conocer en dos etapas, en la primera se presenta la identificación del estado actual del sistema productivo litográfico, que consiste en diagnosticar el proceso mediante la aplicación de un instrumento, encuesta dirigida, diseñada teniendo en cuenta los principales componentes del sistema de producción y la segunda etapa que consiste en la aplicación y análisis del autodiagnóstico de la gestión del sistema productivo (Rajadell,2005), resaltando al final las conclusiones que serán un insumo fundamental para implementar, a futuro, mejoras en el sistema de producción del sector litográfico. 
Durante la primera etapa, se distinguen los elementos del sistema productivo, que pueden constituirse en limitaciones tecnológicas para el sector litográfico, dentro de estos se presentan: la planeación, programación y control de producción, así como la gestión del mantenimiento de maquinaria y equipos.

La Planeación, Programación y Control de la producción, consiste en la planeación de las rutas, la programación, la generación de las órdenes de producción, la coordinación de la inspección, el control de los materiales, las herramientas y los tiempos de las operaciones. El control de producción depende del tipo de producción, por lo cual se puede afirmar que existe una gran variedad de funciones. Según Olavarrieta (1999), para llevar a cabo esto, se requiere de los sistemas de información referentes a: demanda o necesidad de producción, capacidad disponible, tiempos y métodos de las operaciones, disponibilidad de materiales, disponibilidad de herramientas, listas de materiales, progreso o avance en el trabajo, estado y prioridades de las órdenes de producción. De la misma forma, Bello (2006) expone que la función principal de la programación de la producción consiste en el cumplimiento de los planes de producción en lo que respecta a cantidades y fechas, para ello debe mantenerse una adecuada información sobre: maquinaria, materiales, recurso humano.

Por su parte, en la Gestión del mantenimiento, los equipos y las instalaciones se construyen para realizar un trabajo determinado, es evidente que su mantenimiento no es el fin último. Esto implica para el que prioritariamente debe procurar minimizar los problemas que causan dichas averías. Como afirma De Bona (1999) el concepto de mantenimiento está entendido de una forma muy práctica, como lo que hay que hacer para que las cosas funcionen correctamente o, en su defecto, para que las averías duren lo menos posible. Pero este concepto ha evolucionado para formar parte integral del sistema productivo, como se demuestra en la definición expuesta por García (2003), donde el mantenimiento es el conjunto de técnicas destinadas a conservar equipos e instalaciones en servicio durante el mayor tiempo posible, buscando la más alta disponibilidad, y con el máximo de rendimiento.

En la segunda etapa, se aplicó y analizó la situación de la empresa litográfica, desde el punto de vista del mismo empresario, es decir, se realizó un autodiagnóstico. El autodiagnóstico es considerado como el primer paso para la implementación de estrategias o herramientas de mejora continua (Rajadell, 2005). La mejora continua es considerada por los autores como una herramienta necesaria en el funcionamiento correcto de cualquier organización (Rapp y Eklund, 2002), sin discriminación de su tipo de sistema de producción (Bacdayan, 2001; Bond, 1999; Jackson y Dyer, 1998; Schonberger, 1996; Tapping, Luyster y Shuker, 2002).

Los factores objeto de estudio, dentro del autodiagnóstico son seis: Tecnologías y técnicas de diseño y producción, Preparación de máquinas, Productividad, Mantenimiento, Gestión de equipos y Seguridad, orden y limpieza de las instalaciones de producción. Dichos factores fueron seleccionados por pertenecer a áreas donde se pueden presentar limitaciones tecnológicas para el sector litográfico. 


\section{METODOLOGÍA}

La investigación es de tipo estudio de caso, el cual y de acuerdo con Mertens (2005) se considera como la investigación sobre un individuo, grupo, organización, comunidad o sociedad, que es visto y analizado como una entidad. Adicionalmente, instituciones como Harvard Business School (1997) lo considera un método consistente para evaluar unidades organizacionales.

Como se mencionó anteriormente, este documento presenta dos etapas de investigación: la identificación del estado actual y el análisis del autodiagnóstico de la gestión del sistema productivo litográfico.

En la primera etapa, o etapa de identificación del estado actual del sistema productivo, en lo relacionado con tecnología, la unidad de análisis para el estudio de caso la constituyen 19 empresas litográficas de la ciudad de Tunja, registradas en la Cámara de Comercio de la ciudad, las cuales voluntariamente decidieron participar en el proyecto de investigación. La unidad de análisis está constituida por microempresas y pequeñas empresas, puesto que en la ciudad de Tunja, no se encuentran litografías de tamaño mediano o grande.

En la segunda etapa, o etapa de análisis autodiagnóstico, para la unidad de análisis se seleccionaron cuatro empresas, el criterio de selección se determinó teniendo en cuenta lo estipulado en el artículo 2 de la Ley 905 de 2004 del Congreso de Colombia (2004), ver tabla 1, que establece la siguiente clasificación:

\begin{tabular}{|c|c|}
\hline CLASIFICACIÓN & NÚMERO DE EMPLEADOS \\
\hline Microempresa & Menos de 10 \\
\hline Pequeña Empresa & Entre 10 y 50 \\
\hline Mediana Empresa & Entre 51 y 200 \\
\hline Grandes Empresas & Más de 200 \\
\hline
\end{tabular}

Tabla 1. Clasificación de empresas según el número de empleados. Fuente: Congreso de Colombia, 2004.

Las cuatro empresas seleccionadas fueron las ubicadas en el renglón de pequeña empresa, cuentan con las mejores condiciones en infraestructura, activos superiores a 100.000.000 millones de pesos Colombianos y un portafolio diversificado de productos, es decir son las más representativas del sector en la zona bajo estudio y de aquí en adelante se identificarán como empresas litográficas número 1, 2, 3 y 4 . El orden numérico no implica una escala de tipo ordinal, se utilizan los números para etiquetar las empresas seleccionadas. 


\section{Herramientas metodológicas}

Para la primera etapa del trabajo de campo, el instrumento de recolección de información consistió en una encuesta dirigida, la cual incluyó factores a evaluar en el sistema productivo, relacionados directamente con aspectos tecnológicos, como son: planeación, programación y control de la producción y gestión del mantenimiento de maquinaria y equipos (Rodríguez, Balestrini, Meleán, y Rodríguez, 2002). Estos factores fueron examinados mediante revisión bibliográfica, cuyo resultado permitió contrastar autores como: Render y Heizer (1996), Gaither y Frazier (2000), Sipper y Bulfin (2005) y Chase, Jacobs y Aquilano (2009). Adicionalmente, dicha revisión permitió analizar y sistematizar los conceptos relevantes para garantizar el éxito potencial sostenido del proceso productivo en la búsqueda por maximizar la productividad y el aumento de la competitividad en la industria de artes gráficas de la ciudad de Tunja. Los factores claves de evaluación, junto a los elementos evaluados en cada categoría se presentan en la tabla 2.

\begin{tabular}{|l|l|}
\hline \multicolumn{1}{|c|}{ FACTOR CLAVE } & ELEMENTO DE EVALUACIÓN DEL SISTEMA PRODUCTIVO \\
\hline \multirow{2}{*}{$\begin{array}{l}\text { Planeación, Programación } \\
\text { y control de la producción }\end{array}$} & - Método para la programación de la producción. \\
& - Indicadores de productividad para el control de la producción. \\
\hline & - Identificación del cuello de botella. \\
\hline Gestión del & Tiempo de funcionamiento de la maquinaria dentro del pro- \\
mantenimiento & ceso productivo. \\
& - Tipo de mantenimiento realizado. \\
& - Capacitación sobre el mantenimiento de la máquina. \\
\hline
\end{tabular}

Tabla 2. Factores y elementos de evaluación de la situación actual del sistema productivo, relacionados con aspectos tecnológicos.

Durante la segunda etapa del trabajo de campo, se aplicó una encuesta autodiagnóstico dirigida a los representantes del proceso o gerentes de las empresas, obteniendo de esta manera veracidad en la información suministrada. En esta fase, se valoraron seis factores que afectan el sistema productivo de las empresas del sector litográfico, tal como se presenta en la tabla 3. 


\begin{tabular}{|c|c|c|}
\hline FACTOR & PREGUNTA & OPCIONES DE RESPUESTA \\
\hline \multirow{3}{*}{$\begin{array}{c}\text { Tecnologías de } \\
\text { diseño y producción }\end{array}$} & \multirow{3}{*}{$\begin{array}{c}\text { ¿Se utilizan } \\
\text { tecnologías o } \\
\text { técnicas para el } \\
\text { diseño, la mejora, la } \\
\text { diversificación o la } \\
\text { estandarización de } \\
\text { productos? }\end{array}$} & $\begin{array}{l}\text { a) La empresa destaca por unos esfuerzos débiles en } \\
\text { I+D + I y diseño de nuevos productos, cuyos resultados } \\
\text { son difícilmente cuantificables. }\end{array}$ \\
\hline & & $\begin{array}{l}\text { b) La empresa se destaca por unos esfuerzos medios, sobre } \\
\text { los productos existentes, la intención de crear nuevos } \\
\text { productos o diversificar y estandarizar los existentes. }\end{array}$ \\
\hline & & $\begin{array}{l}\text { c) La empresa destaca por unos esfuerzos importantes, } \\
\text { sobre los productos existentes, la creación de nuevos } \\
\text { productos y también sobre el perfeccionamiento de } \\
\text { los procesos de fabricación }\end{array}$ \\
\hline \multirow{3}{*}{$\begin{array}{l}\text { Preparación de } \\
\text { máquinas }\end{array}$} & \multirow{3}{*}{$\begin{array}{l}\text { ¿ Hay preparación } \\
\text { de las máquinas, las } \\
\text { tareas productivas o } \\
\text { el cambio de útiles y } \\
\text { herramientas? }\end{array}$} & $\begin{array}{l}\text { a) No hay ninguna metodología, para la reducción del } \\
\text { tiempo de preparación de las máquinas. Las operaciones } \\
\text { externas y las internas no están diferenciadas. }\end{array}$ \\
\hline & & $\begin{array}{l}\text { b) Se han estandarizado algunas operaciones y las } \\
\text { operaciones de cambio están estandarizadas. Gene- } \\
\text { ralmente, son fácilmente alcanzables las plantillas y } \\
\text { herramientas durante el cambio de utillajes. }\end{array}$ \\
\hline & & $\begin{array}{l}\text { c) Las operaciones internas y externas están separadas } \\
\text { y las operaciones de cambio están organizadas, de ma- } \\
\text { nera que los operarios efectúan cambios de acuerdo } \\
\text { con los manuales. }\end{array}$ \\
\hline \multirow{3}{*}{ Productividad } & \multirow{3}{*}{$\begin{array}{c}\text { En relación al } \\
\text { concepto de pro- } \\
\text { ductividad ¿Qué } \\
\text { puede decirse de la } \\
\text { empresa? }\end{array}$} & $\begin{array}{l}\text { a) No hay ninguna medida cuantitativa de la productividad } \\
\text { o de la rentabilidad económica de la empresa. }\end{array}$ \\
\hline & & $\begin{array}{l}\text { b) La productividad de la empresa permanece estable. } \\
\text { No se consigue incrementar la productividad de una } \\
\text { manera efectiva, incluso habiendo realizado algún tipo } \\
\text { de inversión. }\end{array}$ \\
\hline & & $\begin{array}{l}\text { c) El grado de aprovechamiento de los recursos pre- } \\
\text { senta una tendencia positiva, lo cual indica una mayor } \\
\text { eficiencia productiva. Todos los activos de la empresa } \\
\text { son utilizados y no se dispone de recursos (activos } \\
\text { inmovilizados o circulantes) ociosos. }\end{array}$ \\
\hline
\end{tabular}




\begin{tabular}{|c|c|c|}
\hline FACTOR & PREGUNTA & OPCIONES DE RESPUESTA \\
\hline \multirow[b]{3}{*}{ Mantenimiento } & \multirow{3}{*}{$\begin{array}{l}\text { ¿Qué opción } \\
\text { define mejor su } \\
\text { situación? }\end{array}$} & $\begin{array}{l}\text { a) No existe una política de mantenimiento de las instala- } \\
\text { ciones y las inversiones, aunque quizás sería interesante. }\end{array}$ \\
\hline & & $\begin{array}{l}\text { b) Existe un mantenimiento preventivo por máquina. } \\
\text { Las tareas de mantenimiento se realizan por personal } \\
\text { especializado (interno o subcontratado). }\end{array}$ \\
\hline & & $\begin{array}{l}\text { c) El mantenimiento de las máquinas es esencial. Existen } \\
\text { estadísticas de averías por máquinas o por categorías de } \\
\text { causas, anotando en una ficha las intervenciones (natu- } \\
\text { raleza y fecha). Hay un plan de formación de los operarios } \\
\text { para que puedan realizar operaciones de mantenimiento } \\
\text { básico. Sólo las grandes reparaciones se realizan por } \\
\text { especialistas o empresas subcontratadas. }\end{array}$ \\
\hline \multirow{3}{*}{ Gestión de equipos } & \multirow{3}{*}{$\begin{array}{c}\text { ¿Es posible definir } \\
\text { las grandes líneas } \\
\text { de la política de } \\
\text { inversión a lo } \\
\text { largo de los tres } \\
\text { últimos años? }\end{array}$} & a) No ha habido inversión en los últimos tres años. \\
\hline & & $\begin{array}{l}\text { b) Se ha seguido una política de sustitución y refuerzo } \\
\text { potencial, de manera que la renovación de las instalacio- } \\
\text { nes satisface los objetivos de la producción, en cuanto a } \\
\text { volumen de producción y reducción de costes. }\end{array}$ \\
\hline & & $\begin{array}{l}\text { c) En relación a las inversiones realizadas es posible } \\
\text { precisar y cuantificar los diferentes tipos de objetivos } \\
\text { en términos de mejora de la calidad, reducción de plazos } \\
\text { de entrega y cuellos de botella. }\end{array}$ \\
\hline
\end{tabular}




\begin{tabular}{|c|c|c|}
\hline FACTOR & PREGUNTA & OPCIONES DE RESPUESTA \\
\hline \multirow{6}{*}{$\begin{array}{l}\text { Seguridad, Orden y } \\
\text { Limpieza }\end{array}$} & \multirow{3}{*}{$\begin{array}{l}\text { Después de intentar } \\
\text { visualizar la planta } \\
\text { de producción ¿qué } \\
\text { opción escoge? }\end{array}$} & $\begin{array}{l}\text { a) ¿Porqué limpiar si todo se ensucia de nuevo? En la planta } \\
\text { se acepta la suciedad como condición inevitable de las dis- } \\
\text { tintas estaciones de trabajo. }\end{array}$ \\
\hline & & $\begin{array}{l}\text { b) Los trabajadores de producción asumen que su trabajo es } \\
\text { hacer cosas, no organizarlas o limpiarlas. En algunas áreas de } \\
\text { trabajo, la organización, el orden y la limpieza son las prim- } \\
\text { eras cosas que se dejan de lado cuando hay mucho trabajo } \\
\text { de producción. La explicación es que "estamos demasiado } \\
\text { atareados para ocuparnos de eso". }\end{array}$ \\
\hline & & $\begin{array}{l}\text { c) La organización, el orden, la limpieza, la limpieza } \\
\text { estandarizada y la disciplina han sido el punto de partida } \\
\text { para las actividades de mejora que aseguran la competitivi- } \\
\text { dad y la supervivencia futura de la empresa. }\end{array}$ \\
\hline & \multirow{3}{*}{$\begin{array}{c}\text { ¿Existen } \\
\text { consignas } \\
\text { conocidas por } \\
\text { los empleados } \\
\text { de higiene y } \\
\text { seguridad propias } \\
\text { de la empresa? }\end{array}$} & $\begin{array}{l}\text { a) No todos los operarios conocen las consignas de higiene } \\
\text { y seguridad propias de la empresa, porque los estándares de } \\
\text { seguridad son poco seguidos. }\end{array}$ \\
\hline & & $\begin{array}{l}\text { b) Las medidas de higiene y seguridad están poco desarrol- } \\
\text { ladas. Por ejemplo, no existe un plan de evacuación en caso } \\
\text { de siniestro, ni un control de los niveles de ruido, aunque } \\
\text { paneles de señalización y un plan de localización de los el- } \\
\text { ementos de seguridad (como por ejemplo, los extintores). }\end{array}$ \\
\hline & & $\begin{array}{l}\text { c) Existe un control de utilización de materiales y equipos } \\
\text { individuales de seguridad y protección contra accidentes de } \\
\text { trabajo (ropa, guantes, cascos, gafas, calzados, etc.). }\end{array}$ \\
\hline
\end{tabular}

Tabla 3. Factores de evaluación en el autodiagnóstico de la gestión del sistema productivo

Luego de obtener la información se procedió a su procesamiento y análisis. Se consideraron análisis de carácter cualitativo, realizando una discusión acerca de cada resultado por factor teniendo en cuenta el tipo de respuesta obtenida, como se muestra en la tabla 4.

\begin{tabular}{|c|c|}
\hline $\begin{array}{c}\text { MÉTODO DE } \\
\text { ESTIMACIÓN }\end{array}$ & PORCENTAJE DE USO \\
\hline a) & Sistema productivo tradicional \\
\hline b) & Sistema productivo avanzado \\
\hline c) & Sistema productivo Lean Manufacturing \\
\hline
\end{tabular}

Tabla 4. Categorías de respuesta obtenida. 
La escala de consonantes a, b y c, permite la identificación de la sofisticación del sistema productivo instaurado, es decir, una respuesta tipo a evidencia un sistema productivo menos sofisticado que el de una empresa con respuesta tipo c.

\section{RESULTADOS}

\section{Factores evaluados en el sistema productivo del sector litográfico bajo estudio.}

A continuación se presentan los resultados correspondientes a la primera etapa de investigación, en donde se obtuvieron datos procedentes de las 19 empresas del sector litográfico de Tunja, asociados con los elementos de los sistemas productivos y considerados como limitaciones tecnológicas para este sector.

El primero de los aspectos evaluados en el sistema productivo de las litografías fue la Planeación, Programación y Control de Producción. En este aspecto se evidenció que el 100\% de las litografías de la ciudad de Tunja, no emplean algún tipo de software para la gestión de recursos materiales e inventarios.

Asimismo, la tabla 5 muestra los resultados sobre la manera con que las litografías realizan la estimación de la demanda de sus productos para ejecutar la planeación de actividades de producción.

\begin{tabular}{|c|c|}
\hline MÉTODO DE ESTIMACIÓN & PORCENTAJE DE USO \\
\hline Empírico & 79 \\
\hline Ninguno & 16 \\
\hline Pronósticos & 5 \\
\hline
\end{tabular}

Tabla 5. Método de estimación de la demanda en litografías de Tunja.

Es evidente que el cálculo o estimación de la demanda como insumo fundamental para la planificación de la producción en las litografías se realiza de manera empírica, es decir, la demanda se estima a partir de la experiencia del empresario y de algunos datos históricos sobre las ventas. Además, es notable el bajo uso en el método de pronósticos para la estimación de la demanda que el empresario de este sector aplica al interior de la empresa, en su mayoría esto se debe al desconocimiento de la técnica para el cálculo de pronósticos. 
Otro de los aspectos evaluados fueron los criterios para la programación de actividades productivas, esto corresponde a la manera con que la empresa programa los pedidos del cliente una vez llegan al piso de producción. La tabla 6 presenta los resultados obtenidos del sector bajo estudio para el aspecto programación de actividades productivas.

\begin{tabular}{|c|c|}
\hline CRITERIO PARA LA PROGRAMACIÓN & PORCENTAJE DE USO \\
\hline Prioridad a ciertos clientes & 63 \\
\hline Primeros en llegar & 16 \\
\hline Tiempos de entrega más cortos & 16 \\
\hline No sabe o No responde & 5 \\
\hline
\end{tabular}

Tabla 6. Criterios para la programación de actividades productivas.

Los resultados obtenidos en los criterios utilizados para programar las actividades productivas evidenciaron que la prioridad al momento de atender los pedidos de los diferentes clientes consiste en clasificar y dar celeridad a ciertos clientes que las empresas consideran como especiales, es decir aquellos clientes que compran con mayor frecuencia y cantidad. Luego de la programación de pedidos a clientes especiales, se encontró la asignación de trabajos siguiendo la regla primeros en llegar, primeros en salir y aquellos pedidos con tiempos de entrega más cortos.

De otra parte, el 68\% de las empresas litográficas no cuentan con sistemas de indicadores de gestión, especialmente relacionados con la productividad. En ese mismo sentido, las litografías encuestadas identifican sus cuellos de botella, recursos que limitan la capacidad del sistema, en tres puntos importantes del proceso, como se observa en la figura 1.

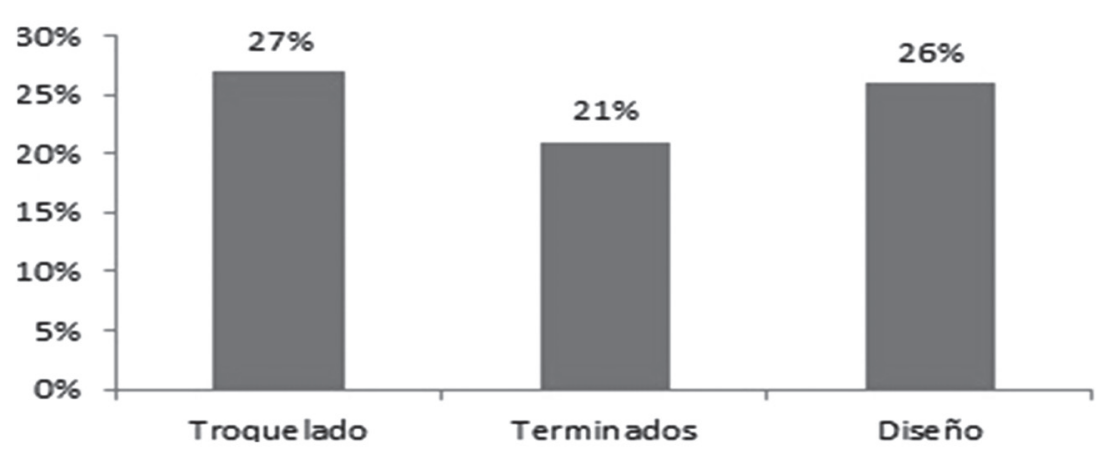

Figura 1. Procesos que generan cuellos de botella 
En relación con la gestión del mantenimiento, tecnología y equipos, los hallazgos son los siguientes: se evidenció que la edad de la maquinaria en las empresas litográficas bajo estudio es superior a 15 años (63\% de los casos), dado que son empresas con una trayectoria importante en la ciudad de Tunja y en varios casos obtuvieron maquinaria usada. Por otra parte, el mantenimiento realizado en la mayoría de estas empresas es de tipo preventivo y correctivo ( $53 \%$ de los casos) y el porcentaje restante (43\% de los casos) no involucra a sus empleados cuando se requiere hacer mantenimiento, de hecho sólo aplican mantenimiento correctivo cuando la maquinaria presenta alguna falla.

\section{Gestión actual del sistema productivo litográfico.}

La tabla 7 muestra el resumen de las respuestas obtenidas de las cuatro empresas en cada factor evaluado, tal como se menciona en la etapa dos de la metodología. Las empresas se encuentran en las columnas, numeradas consecutivamente de uno a cuatro, los factores evaluados se ubican en las filas de la tabla, y en su interior las respuestas obtenidas sean del tipo a, b o c.

\begin{tabular}{|c|c|c|c|c|}
\hline FACTORES - EMPRESA & 1 & 2 & 3 & 4 \\
\hline Tecnologías y técnicas de diseño y producción & c & c & c & b \\
\hline Organización de la producción & a & b & b & b \\
\hline Productividad & c & c & c & c \\
\hline Mantenimiento & b & c & b & b \\
\hline Gestión del equipo productivo & b & c & b & b \\
\hline Seguridad, orden y limpieza & c & b & b & c \\
\hline
\end{tabular}

Tabla 7. Respuestas obtenidas de los factores por empresa

\section{DISCUSIÓN DE RESULTADOS}

De acuerdo con los resultados obtenidos en la primera etapa de la investigación, es evidente que en el primer aspecto evaluado sobre planeación, programación y control de producción en las litografías, no existe el uso de software para este propósito, lo cual indica que existe una falencia en el manejo de herramientas adecuadas para planificar de manera coherente la producción y obtener resultados alineados con las necesidades del cliente. De hecho, los resultados confirman que ninguna de las empresas bajo estudio, emplean software para la gestión de recursos materiales e inventarios, quizás por el nivel educativo de la fuerza de trabajo o por la capacidad económica de estas empresas. 
Al mismo tiempo, la no utilización de herramientas para la estimación de la demanda de sus productos, convierten a la industria litográfica en agente vulnerable a cambios del entorno, pese a la existencia de diversas técnicas y tecnologías que actualmente existen para la gestión del sistema productivo. El uso de la experiencia si bien es un argumento válido para estimar la demanda, deja un alto grado de incertidumbre sobre las necesidades del cliente, lo cual impactará en la correcta planificación y programación de la producción.

Por su parte, la programación de los trabajos que provienen de los diferentes clientes, se clasifican según el tipo de cliente, esto es, si es un cliente especial, se atiende primero su solicitud y luego se atienden a los demás clientes de acuerdo con el orden de llegada o según el tiempo de elaboración de los productos. En tal sentido, los clientes especiales seguirán comprando a estas litografías debido a su especial atención. Sin embargo, los pequeños compradores o clientes nuevos se pueden ver afectados en el escenario en que deban esperar a que la litografía primero procese un pedido de un cliente especial, para que luego se atendido su pedido.

La ausencia en el uso y aplicación de indicadores en el sistema productivo de las litografías es un aspecto a mejorar, dado que al no medir el desempeño del proceso productivo, se dificulta controlarlo y por ende establecer acciones de mejora sobre el mismo.

En cuanto a la realización del mantenimiento, la mayoría de las industrias del sector litográfico prefiere contratar a personal externo capacitado y con experiencia en el manejo de maquinaria y equipos propios del sector, debido a que en el proceso de autodiagnóstico de los empresarios, se evidenció la necesidad de contar con personal capacitado en las labores de la industria, teniendo presente, que el mercado laboral no cuenta con formación especializada en el área técnica de las litografías, especialmente en el manejo y mantenimiento de sus equipos.

\section{Tecnologías y técnicas de diseño y producción}

En este aspecto, tres empresas estudiadas presentaron una respuesta tipo c, significa entonces que el área de diseño en estas empresas es apoyada por los departamentos que tienen relación directa con la calidad del producto, destacándose como ventajas el trabajo en equipo, mayor capacidad para tomar iniciativas y la formación de especialistas con un horizonte más amplio y con especial sensibilidad a las necesidades y condiciones del mercado. Al permitir que las ideas del diseño sean simultáneas a las necesidades y expectativas de los clientes se reduce el período de desarrollo contribuyendo al éxito comercial, reduciendo costos y elaborando un producto de alta calidad. Es preciso destacar que el diseño es una de las etapas críticas del proceso de producción litográfico, por esta razón, es notoria la preocupación de los directivos por implementar mejoras en dicha etapa. 


\section{Organización de la producción}

Nuevamente, tres de las litografías estudiadas presentaron una respuesta del tipo b, mostrando una planificación centralizada y compleja, haciendo una detallada supervisión en el uso de los recursos. Este tipo de organización se puede clasificar como clásica, debido a que la producción en cada puesto de trabajo se planifica sobre la base de unas previsiones de venta y de los stocks de productos terminados. Dentro del control de la producción resulta prácticamente imposible examinar todas las situaciones relacionadas con la velocidad de la producción y nivel de existencias. No se pueden efectuar mejoras respecto al tamaño de lote y sincronización de procesos, porque resulta demasiado engorroso calcular detalladamente los planes óptimos de producción.

\section{Productividad}

En este aspecto, las empresas analizadas coinciden en responder que el incremento de la productividad es el resultado de trabajar con mayor rapidez, y la implementación de métodos eficientes de trabajo (Rajadell, 2005). Las prioridades de cumplir los requisitos del cliente, dejan de lado la medición de los índices de mejora de productividad como la relación costo-beneficio de los trabajos realizados.

\section{Mantenimiento}

Las litografías 1, 3 y 4 realizan procedimientos de mantenimiento similares, en donde las inversiones se asocian con la compra de equipos grandes, caros y que trabajan a gran velocidad, tal y como lo expresa Rajadell (2005). Asimismo, se evidencia que las empresas se encuentran más pendientes del funcionamiento de los equipos que del cliente.

Por otra parte, la litografía 2 se distingue por sus inversiones en maquinaria y equipo, lo cual permite que la fabricación alcance las metas de costos, calidad, rendimiento del producto, entrega, flexibilidad e innovación (Rajadell, 2005). La adquisición de mayor tecnología le exige mantener elevadas tasas de utilización y la eficiencia de toda la compañía.

\section{Gestión de equipos}

En este aspecto, la divergencia de opiniones de los gerentes o directores de las litografías no permite obtener un comportamiento marcado en el sector. Sin embargo, a continuación se presentan algunas características de sus sistemas de gestión de equipos.

Las litografías 1, 3 y 4, presentan un sistema clásico en gestión del mantenimiento, donde no se involucra a los operarios directos de las máquinas. El mantenimiento de los equipos es realizado por personal externo a la empresa. En cuanto a la reposición de equipos y maquinaria, estas empresas se caracterizan por invertir sólo cuando se requiere reemplazar o mejorar las máquinas. No se evidencia mantenimiento de tipo proactivo. 
Por su parte, la litografía 2 se enmarca en el concepto de Mantenimiento Productivo Total (TPM), el cual se apoya en el conocimiento de los operarios respecto al funcionamiento de la máquina. Gracias a este sistema, los operarios pueden identificar a tiempo problemas de la maquinaria mediante el control de ruidos, desgastes o vibraciones no habituales.

\section{Seguridad, orden y limpieza}

Las litografías 1 y 4 se destacan por sus actividades de limpieza, orden y aseo en oficinas y talleres. Lo anterior, permite que estas empresas mejoren su calidad, disminuyan algunos costos y mejoren su relación con los clientes. Adicionalmente, un ambiente de trabajo adecuado facilita las comunicaciones y las relaciones de los empleados. La distribución de la planta permite la agilidad en los procesos.

Entre tanto, las litografías 2 y 3 no consideran importante mantener el aseo, orden y limpieza de los espacios, viendo este aspecto como algo secundario, lo que hace que el proceso productivo presente falencias de acuerdo a la falta de organización en las estaciones de trabajo, generando a la vez, un clima organizacional poco agradable para el personal operativo de la empresa, lo que dificulta las relaciones con los empleados, presentando demoras en el proceso productivo.

\section{CONCLUSIONES}

Como resultado de la no utilización de software o técnicas actualizadas para la planeación, programación y control de producción, el sistema de producción de las diferentes empresas litográficas se encuentra amenazado por el atraso tecnológico, dado que, el empirismo se encuentra presente al momento de planear las operaciones en estas empresas. Aspecto que se encuentra en contravía con el desarrollo tecnológico que exige un sector competitivo como es el de la industria de artes gráficas. De igual manera, el hallazgo de solo cuatro empresas de tamaño pequeño y con perspectivas de crecimiento futuro, expone un problema industrial en crecimiento para la ciudad.

La gestión del sistema productivo repercute directamente sobre la calidad en varios aspectos: el servicio, el producto y la gestión de los procesos. Por lo tanto, las empresas litográficas priorizan la calidad final del producto, desconociendo el control de calidad durante cada fase de la elaboración del mismo. Lo cual implica una alta generación de defectos o desperdicio de producto, debido a que, si únicamente se realiza control de calidad al finalizar el producto, aquellos que resulten defectuosos tendrán que ser reelaborados o desechados. En este caso las pérdidas y la ineficiencia del proceso hacen que las litografías de la ciudad de Tunja pierdan competitividad dentro del sector nacional.

Por otra parte, el autodiagnóstico del sistema productivo es una herramienta útil, que permite a los gerentes y jefes de proceso obtener una mirada interna y concreta acerca de la forma como se gestiona el sistema productivo, examinando diferentes factores como el estilo de dirección, tecnologías y técnicas de diseño y producción, organización de la producción, productividad, mantenimiento y seguridad, orden y limpieza. 
En consecuencia, el autodiagnóstico realizado en las cuatro empresas más representativas de la ciudad de Tunja evidenció que en su mayoría las respuestas más comunes son del tipo b), lo cual implica que los gerentes de estas empresas y en coherencia con lo planteado por Rajadell (2005), trabajan para liderar, guiar y coordinar el rumbo de sus empresas, orientados hacia el logro de resultados, mostrando interés por compartir utilidades con los inversores, proveedores y empleados. Sin embargo, los factores organización de la producción, mantenimiento y gestión del equipo productivo, deben mejorarse desde la gestión realizada por parte de la gerencia. Las mejoras pueden obtenerse a través de la aplicación de herramientas para la mejora continua, que logren dar solución a los problemas evidenciados en cada uno de dichos factores.

Los cambios imprevisibles de la demanda se han convertido en una limitación para la adecuada planeación de la producción, sin embargo, tal situación no puede convertirse en una excusa para la ineficiencia de los procesos. Por tal razón, y en vías de la implementación de programas de mejora continua, se deben revisar las estrategias de producción encaminadas a mejorar los pronósticos de ventas, que minimicen las variaciones entre las ventas reales y esperadas. A su vez, el adecuado pronóstico de las ventas mejorará la gestión de los inventarios de materias primas y productos terminados.

De igual forma, la implementación de un sistema de indicadores para la medición de la productividad en las empresas litográficas, se convierte en otro de los elementos sugeridos para iniciar un programa de mejora continua. Mediante el autodiagnóstico, se evidenció que los administradores del proceso utilizan su criterio personal, basado en la experiencia, para medir la productividad de su sistema de producción, por lo cual, desconocen elementos importantes de la productividad, e incluso en la gestión de equipos y personal de producción.

Finalmente, el análisis realizado demuestra la necesidad de las empresas del sector litográfico de incrementar su capacidad tecnológica para ser más competitivas en el mercado. Sin embargo, las mediciones del diagnóstico realizado a la gestión del sistema productivo, expone limitaciones en cuanto al acceso de capital y la fuerte competencia generada por empresas de la capital del país. 


\section{REFERENCIAS BIBLIOGRÁFICAS}

Alcaldía de Tunja. (2010). Directorios Alcaldía y Entidades: Entidades dedicadas a la litografía. Recuperado el 3 de Diciembre de 2010, de Oficina de Comunicaciones y Prensa: www.tunja.gov.vo/?dcategoria=8508\#

Bacdayan, P. (2001). Quality improvement teams that stall due to poor project selection: an exporation of contributing factors. Total Quality Management, 12(5), 589-598.

Bello, C. (2006). Manual de producción aplicado a las PYME. Bogotá, ECOE ediciones.

Bond, T. (1999). The role of performance measurement in continuous improvement. International Journal of Operations \& Production Management, 19(12), 13-18.

Cámara de Comercio de Tunja. (2010). Base de datos clasificada empresas de litografía, tipografía y afines.Tunja.

Congreso de Colombia. (2 de Agosto de 2004). Por medio de la cual se modifica la Ley 590 de 2000 sobre promoción del desarrollo de la micro, pequeña y mediana empresa colombiana y se dictan otras disposiciones. Ley 905 de 2004. Bogotá.

Chase, R., Jacobs, R., y Aquilano, N. (2009). Administración de operaciones, producción y cadena de suministros. México: McGraw-Hill.

De Bona, J. (1999). La gestión del mantenimiento: Guía para el responsable de la conservación de locales e instalaciones; criterios para la subcontratación. Madrid, FC editorial.

Gaither, N., \& Frazier, G. (2000). Administración de producción y operaciones. México: Cengage Learning Editores.

García, S. (2003). Organización y gestión integral de mantenimiento. España. Editorial Díaz de Santos.

Harvard Business School, (1997). Case Study Program guidelines. Boston, MA, EE. UU.: Harvard University, Harvard Business School Publishing and Design Management Institute Press.

Jackson, T., y Dyer, C. (1998). Diagnóstico corporativo: una herramienta para alcanzar la excelência (Primera ed.). Madrid: TGP Hoshin (PRODUCTIVITY PRESS).

Mertens, D. (2005). Research and evaluation in Education and Psychology: Integrating diversity with quantitative, qualitative, and mixed methods. Thousand Oaks: Sage. 
Rajadell, M. (2005). La primera fase para la implantación del proceso de mejora continua: el autodiagnóstico del sistema productivo de la empresa. Recuperado el 12 de Septiembre de 2010, de Universidad Politécnica de Cataluña: http://upcommons.upc.edu/e-prints/bitstream/2117/505/1/

Rapp, C., y Eklund, J. (2002). Sustabinable development of improvement activities: The long-term operation of a suggestion scheme in a Swedish company. Total Quality Management, 13(7), 945-969.

Render, B., \& Heizer, J. (1996). Principios de Administración de Operaciones. México: McGraw-Hill.

Rodríguez, G., Balestrini, S., Balestrini, S., Meleán, R., y Rodríguez, B. (2002). Análisis estratégico del proceso productivo en el sector industrial. Revista de Ciencias Sociales, VIII, 135-156.

Schonberger, R. (1996). World Class Manufacturing: the next decade.New York: Free Press.

Sipper, D., y Bulfin, J. (2005). Planeación y control de la Producción.México: McGraw-Hill.

Olavarrieta, J. (1999). Nociones de control de producción, costos, suministros e inventarios - conceptos generales de productividad. España, Editorial Universidad Iberoamericana.

Tapping, D., Luyster, T., y Shuker, T. (2002). Value Stream management eight steps to planning, mapping, and sustaining lean improvements (Primera ed.). New York: Productivity Press. 